\title{
Angiossarcoma de cabeça e pescoço do idoso: um relato de caso
}

\section{Angiosarcoma of the head and neck of the elderly: a case report}

Érika Belizário Soares ${ }^{1}$. Emily Mourão Soares Lopes ${ }^{1}$. Érica Bezerra de Sena ${ }^{1}$. Kalina Ribeiro Fontenele Bezerra $^{1}$. Amanda Maria Menezes Dantas ${ }^{1}$. Alinne Mota Cavalcante ${ }^{1}$. José Wilson Accioly Filho ${ }^{1,2}$.

1 Universidade Federal do Ceará (UFC), Fortaleza, Ceará, Brasil. 2 Hospital Universitário Walter Cantídio (HUWC/UFC/ EBSERH) Fortaleza, Ceará, Brasil.

\section{RESUMO}

O angiossarcoma é uma neoplasia vascular incomum, agressiva, de apresentação clínica variável e difícil diagnóstico, apresentando altas taxas de recidiva local, metástases precoces e prognóstico ruim. O angiossarcoma envolvendo face e couro cabeludo de pacientes idosos é um subtipo raro, acomete mais comumente homens do que mulheres e é discretamente mais prevalente em pacientes caucasianos. O tratamento de escolha é a ampla excisão cirúrgica, sendo a radioterapia e a quimioterapia opções terapêuticas, dependendo da idade do paciente e da extensão local. O presente relato de caso trata-se de uma paciente com angiossarcoma extenso de cabeça e pescoço, de rápida evolução, tendo-se optado por tratamento quimioterápico, devido extensão do quadro.

Palavras-chave: Hemangiossarcoma. Neoplasias vasculares. Cabeça. Pescoço. Quimioterapia.

\section{ABSTRACT}

Angiosarcoma is an uncommon, aggressive vascular neoplasm with variable clinical presentation and difficult diagnosis, presenting high rates of local recurrence, early metastasis and poor prognosis. Angiosarcoma involving the face and scalp of elderly patients is a rare subtype, it affects more commonly men than women and it is discretely more prevalent in Caucasian patients. The treatment of choice is extensive surgical excision, with radiotherapy and chemotherapy being therapeutic options, depending on the patient's age and local extent. The present case report is a patient with extensive angiosarcoma of head and neck, of rapid evolution, having opted for chemotherapy treatment, due to extension of the condition.

Keywords: Hemangiosarcoma. Vascular neoplasms. Head. Neck. Chemotherapy.

Autor correspondente: Érika Belizário Soares, Coronel Nunes de Melo, sem número, Rodolfo Teófilo. Fortaleza, Ceará. CEP: 60416-000. Telefone: +55 85 99135-5956. E-mail: erikabelizario@hotmail.com

Conflito de interesses: Não há qualquer conflito de interesses por parte de qualquer um dos autores.

Recebido em: 02 Fev 2019; Revisado em: 25 Dez 2020; Aceito em: 05 Mai 2021. 


\section{INTRODUÇÃO}

O angiossarcoma é uma neoplasia vascular rara, proveniente de células endoteliais, responsável por menos de $2 \%$ dos sarcomas de partes moles e menos de $1 \%$ de todas as neoplasias de cabeça e pescoço. ${ }^{1} \mathrm{O}$ angiossarcoma cutâneo é um tumor altamente maligno e é subdivido em três tipos clínicos: angiossarcoma da cabeça e pescoço do idoso; angiossarcoma associado ao linfedema crônico e angiossarcoma pós-irradiação. ${ }^{2} \mathrm{O}$ angiossarcoma da cabeça e pescoço do idoso foi descrito pela primeira vez por WilsonJones, é geralmente limitado à pele e tecidos moles e acomete mais comumente homens $(2: 1)$ entre a sétima e oitava década de vida, sendo discretamente mais prevalente em pacientes caucasianos. ${ }^{1,2,3}$ Sua apresentação varia de uma pequena placa a nódulos multifocais, podendo se apresentar de diversas formas, simulando doenças infecciosas e inflamatórias dificultando o diagnóstico precoce. O angiossarcoma é um tumor muito agressivo com altas taxas de recidiva local, metástases precoces e de mau prognóstico. ${ }^{1}$

\section{RELATO DE CASO}

Paciente, 89 anos, sexo feminino, sem comorbidades prévias, apresentando história de 5 meses de evolução de surgimento de placas eritematovioláceas, infiltradas, inicialmente em região periorbitária, com rápida progressão para região malar, fronte e couro cabeludo, associada a alopécia difusa acometendo principalmente na região parietal e de vértice. (Figuras 1, 2 e 3) Referia astenia e perda de peso discreta não mensurada. Negava história familiar de doenças neoplásicas. Exames laboratoriais evideciaram hemoglobina de $10 \mathrm{~g}$ / dL com padrão normocítico e normocrômico, leucograma e plaquetograma sem alterações; função renal, tireoidiana e nível de transaminases estavam dentro do limite de normalidade; FAN e fator reumatoide foram não reagentes. Seguiu-se investigação com biópsia de pele em 3 pontos (couro cabeludo e regiões malares direita e esquerda) que evidenciou neoplasia constituída por proliferação de espaços vasculares irregulares revestidos por células endoteliais hipercromáticas, com moderado pleomorfismo nuclear, dissecando as fibras colágenas ao longo de toda a derme. (Figuras 4 e 5)

Estudo imunohistoquímico mostrou positividade para CD 31, CD 34, ERG e Ki-67 em 20\% das células neoplásicas e negatividade para HHV 8 e citoceratina, sendo o quadro compatível com angiossarcoma grau 2. Estudos de imagem de tórax e abdome não evidenciaram alterações. Devido extensão do tumor e impossibilidade cirúrgica, paciente foi então qualificada para tratamento quimioterápico com paclitaxel.

Nossa paciente realizou seis ciclos de quimioterapia, apresentando melhora do aspecto das lesões, porém, com mielotoxicidade importante, com consequente suspensão do tratamento. Seguiu com tratamento paliativo acompanhada pela Oncologia clínica, apresentando progressão da doença (Figura 6) e agravamento do quadro clínico, vindo a óbito alguns meses depois.
Figura 1. Placas eritematovioláceas, infiltradas acometendo região periorbitária, malar e frontal.

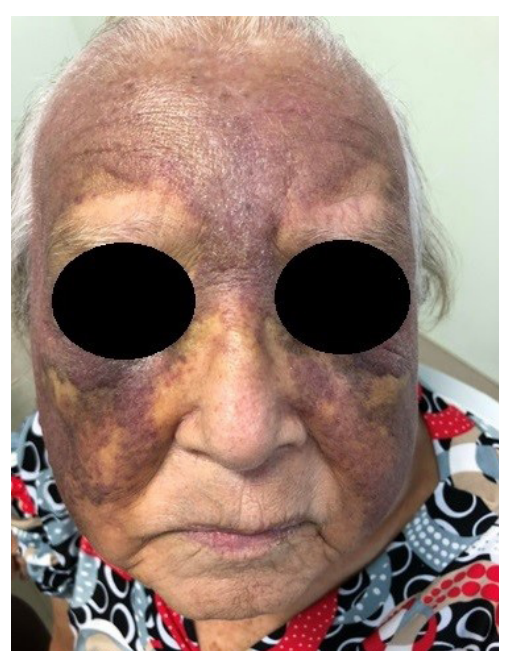

Figura 2. Placas eritematovioláceas, infiltradas acometendo região periorbitária, malar, frontal e couro cabeludo com área de alopecia difusa.

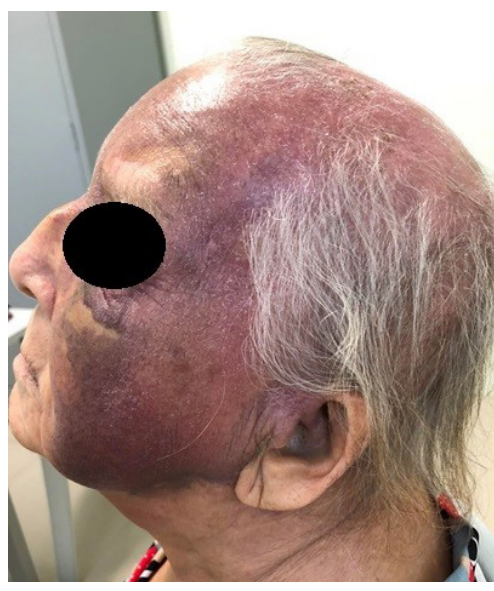

Figura 3. Placas eritematovioláceas acometendo região de couro cabeludo, com área de descamação e alopecia principalmente em região parietal e de vértice.

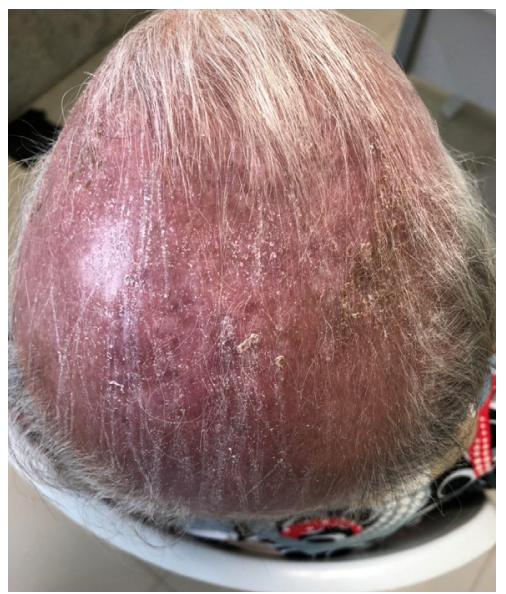


Figura 4. Fragmento de pele apresentando neoplasia constituída por proliferação de espaços vasculares irregulares que dissecam as fibras colágenas ao longo da derme (HE,40X).

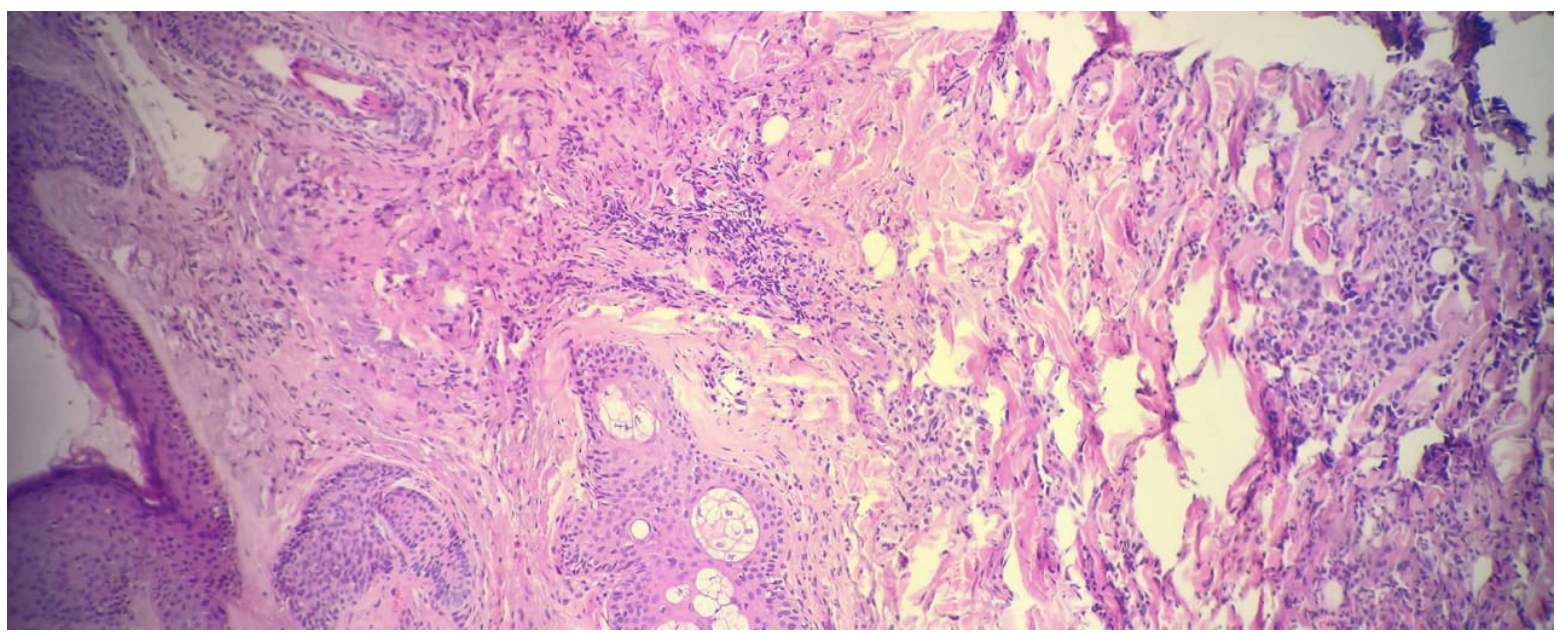

Figura 5. Revestimento de espaços vasculares constituído por células hipercromáticas, com moderado pleomorfismo nuclear. (HE, 400X).

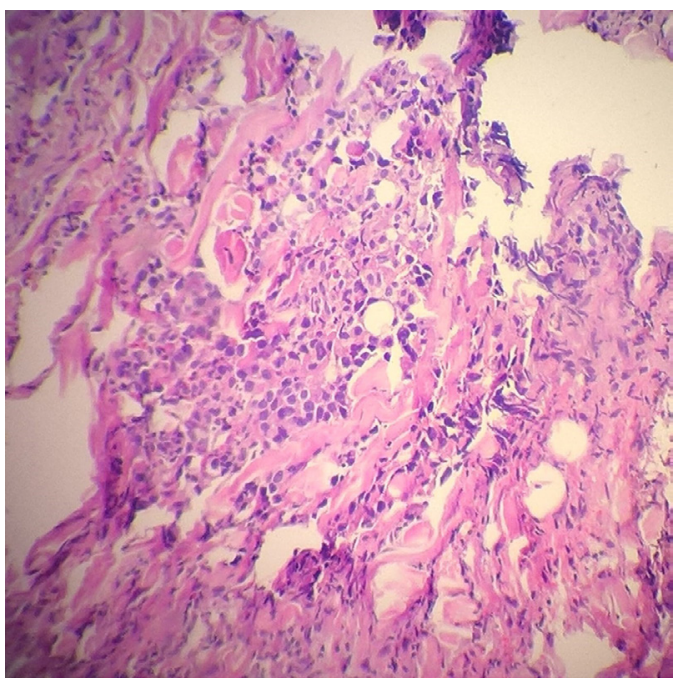

Figura 6. Placas e nódulos eritematosos infiltrados em face, evidenciando piora do aspecto das lesões após suspensão do tratamento.

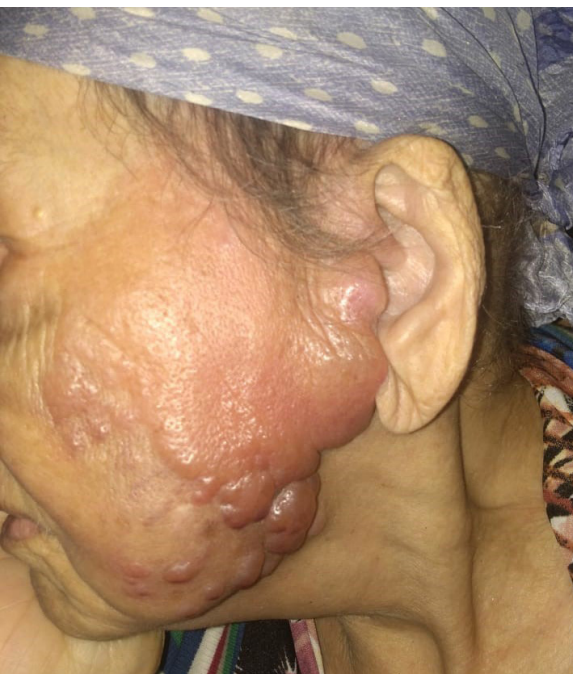

\section{DISCUSSÃO}

Angiossarcomas cutâneos são sarcomas raros de partes moles, podendo ser agrupados com base em características macroscópicas ou histológicas. Clinicamente, a maioria dos pacientes apresenta uma mácula ou nódulo de aspecto equimótico, podendo apresentar placas eritematosas endurecidas, ulcerações e até sangramento. Lesões ulceradas e hemorrágicas indicam doença avançada. ${ }^{1,4}$ Inicialmente, eles podem simular várias outras doenças, como rosácea, hematomas, celulite e angioedema facial dificultando seu diagnóstico. ${ }^{5,6}$

O fato incomum do presente caso foi o desenvolvimento de extensa área de alopecia na região de couro cabeludo acometida pelo tumor. Alopecia é uma apresentação incomum, com poucos casos relatados na literatura e parece estar relacionada a tumores mal diferenciados. ${ }^{4,7} \mathrm{Knight}$ et al. relataram um caso raro em que o angiossarcoma do couro cabeludo apresentava extensa alopecia cicatricial e Murray et al. descreveram um caso de alopecia significativa com miniaturização de pelos em um angiossarcoma de couro cabeludo. ${ }^{4,7}$

Linfedema crônico, exposição à radioterapia prévia, tratamento de carcinomas mamários e imunossupressão em pacientes transplantados renais estão associados ao desenvolvimento de angiossarcomas. No entanto, ainda pouco se sabe com relação aos fatores causais dessa doença e, na maioria dos casos, nenhum fator predisponente é encontrado., ${ }^{3,6}$ Quanto à patogênese, a regulação positiva do glicopeptídeo VEGF-D (um fator de crescimento endotelial vascular), dentre outros, parece ser responsável pela proliferação de células endoteliais. ${ }^{6}$ 
Histologicamente, os tumores contêm uma proliferação de canais vasculares ramificados que "dissecam" estruturas adjacentes. É observado um aumento do número e do tamanho de células endoteliais que revestem os canais vasculares; podendo estas ter formato esférico, epitelioide ou poligonal. ${ }^{2}$ Sua classificação varia de baixo a alto grau, dependendo de sua celularidade, pleomorfismo e atividade mitótica. Os angiossarcomas de baixo grau são bem diferenciados, enquanto as lesões de alto grau são pouco diferenciadas e consistem em lâminas de células pleomórficas com áreas de hemorragia, arquitetura desordenada, células com núcleos hipercromáticos e pleomórficos com atividade mitótica proeminente. Ambos os tipos estão associados a um crescimento local extenso, envolvendo a derme microscopicamente, podendo invadir estruturas mais profundas, no caso de tumores mal diferenciados. $^{2}$

O diagnóstico diferencial histopatológico inclui alguns tipos de hemangiomas, carcinoma acantolítico e sarcoma de Kaposi. ${ }^{3,6,8}$ Apesar de não patognomônica, a positividade imunohistoquímica para os marcadores endoteliais CD 31, CD 34 e antígeno relacionado ao fator VIII podem ajudar a estabelecer o diagnóstico. .,6,9 $^{3}$

Metástases à distância podem ocorrer em até 50\%, sendo o pulmão o local mais comum, seguido por linfonodo, osso e fígado. ${ }^{8}$ A natureza altamente metastática desses tumores tem sido atribuída à ausência de caderina endotelial vascular (VE-caderina), que está presente no endotélio normal. $^{2}$

Vários fatores estão supostamente associados ao pior prognóstico da doença sendo os principais: diâmetro do tumor maior que $5 \mathrm{~cm}$, profundidade de invasão $(>3 \mathrm{~mm})$, status de

\section{REFERÊNCIAS}

1. Mullins B, Hackman T. Angiosarcoma of the head and neck. Int Arch Otorhinolaryngol. 2015;19(3):191-5.

2. DasGupta M, Chakrabarti N, Agrawal P, Narurkar S. Angiosarcoma of the scalp. Indian J Plast Surg. 2009;42(1):118-21.

3. Glickstein J, Sebelik ME, Qing Lu. Cutaneous angiosarcoma of the head and neck: a case presentation and review of the literature. Ear Nose Throat J. 2006;85(10):672-4

4. Murray S, Simmons I, James C. Cutaneous angiosarcoma of the face and scalp presenting as alopecia. Australas J Dermatol. 2003;44:273-6.

5. Ambujam S, Audhya M, Reddy A, Roy S. Cutaneous angiosarcoma of the head, neck, and face of the elderly in type 5 skin. J Cutan Aesthet Surg. 2013;6(1):45-7. margem positivo, couro cabeludo como localização primária, presença de metástase à distância e recorrência. ${ }^{8,9}$

O angiossarcoma cutâneo é de difícil tratamento e o consenso atual é a excisão ampla da lesão, associada, na maioria das vezes, à radioterapia ou quimioterapia adjuvantes. A obtenção de margens histologicamente livres de tumor afeta diretamente no prognóstico da doença, no entanto, devido ao espaço relativamente pequeno na cabeça e no pescoço, à proximidade de estruturas vitais e ao extenso padrão microscópico de disseminação vascular destes tumores, pode ser difícil obter margens negativas, levando à altas taxas de recidiva local após a excisão cirúrgica, o que corresponde entre 35 a $86 \%$ dos casos na literatura. E apesar dos conceitos de terapia multimodal, o prognóstico permanece insatisfatório. ${ }^{1}$

No referido caso, a excisão cirúrgica não era uma opção terapêutica devido à grande extensão tumoral, sendo optado tratamento quimioterápico com paclitaxel, um agente com efeito de indução antiangiogênica e apoptótica, inibindo seletivamente a proliferação de células endoteliais. ${ }^{4}$ Este, quando usado isoladamente parece ser útil em angiossarcomas de face e couro cabeludo. ${ }^{5}$

\section{CONCLUSÃO}

Em resumo, o presente caso de angiossarcoma de face e couro cabeludo demonstra uma neoplasia agressiva de rápida progressão, elucidando a importância do diagnóstico precoce e a dificuldade terapêutica em casos de doença extensa. Desse modo, em todos os pacientes idosos com lesões no couro cabeludo e na região da cabeça e pescoço, a possibilidade de angiossarcoma deve ser lembrada para evitar atrasos desnecessários no tratamento.

6. Ettl T, Kleinheinz J, Mehrotra R, Schwarz S, Reichert TE, Driemel O. Infraorbital cutaneous angiosarcoma: a diagnostic and therapeutic dilemma. Head Face Med. 2008;4:18.

7. Knight TE, Robinson HM, Sina B Júnior. Angiosarcoma (Angioendothelioma) of the scalp: an unusual case of scarring Alopecia. Arch Dermatol. 1980;16:683-6.

8. Fujisawa Y, Yoshino K, Fujimura T, Nakamura Y, Okiyama N, Ishitsuka Y, et al. Cutaneous angiosarcoma: the possibility of new treatment options especially for patients with large primary tumor. Front Oncol. 2018;8:46.

9. Morgan MB, Swann M, Somach S, Eng W, Smoller B. Cutaneous angiosarcoma: a case series with prognostic correlation. J Am Acad Dermatol. 2004;50(6):867-74.

\section{Como citar:}

Soares EB, Lopes EM, Sena EB, Bezerra KR, Dantas AM, Cavalcante AM, et al. Angiossarcoma de cabeça e pescoço do idoso: um relato de caso. Rev Med UFC. 2021;61(1):1-4. 